\title{
Efeitos de Forças Gravitacionais e Não-Gravitacionais sobre o Movimento Orbital de Satélites Artificiais
}

\section{Effects of Gravitational and Non-Gravitational Forces on the Orbital Motion of Artifitial Satellites}

\author{
Jean Paulo dos Santos Carvalho* e Rodolpho Vilhena de Moraes $^{\dagger}$ \\ Grupo de Dinâmica Orbital e Planetologia - UNESP, \\ Av. Ariberto Pereira da Cunha, 333 \\ Guaratinguetá - SP - 12516-410
}

\begin{abstract}
Uma teoria para estudar o movimento orbital de satélites artificiais sobre efeitos do arrasto atmosférico e da pressão de radiação solar direta - considerando a sombra da Terra e alguns termos do geopotencial - é desenvolvida analiticamente. A sombra da Terra é modelada utilizando a função sombra $(\psi)$, como introduzida por Ferraz Mello: $\psi$ igual zero quando o satélite está na região de sombra e igual a um quando é iluminado pelo Sol. As componentes do arrasto são dadas por Vilhena de Moraes baseado no modelo atmosférico TD-88. O método de Hori para sistemas não-canônicos é aplicado para resolver as equações de movimento. Um software para manipulação algébrica é fundamental para fazer os cálculos necessários. Efeitos seculares e periódicos que influenciam no movimento orbital de satélites artificiais são analisados. É dada ênfase aos termos de acoplamento que surgem na solução do sistema de equações diferenciais. Utilizando dados orbitais do satélite CBERS-1 é feito um estudo para analisar ordens de grandeza da variação do semi-eixo maior devidas às perturbações consideradas.
\end{abstract}

Palavras-chaves: Teoria de Perturbação, Arrasto Atmosférico, Pressão de Radiação Solar Direta, Satélites Artificiais.

A theory to study the orbital motion of artificial satellites under the effects of the atmospheric drag and of the direct solar radiation pressure - considering the Earth's shadow and some terms of the geopotential - is analytically developed. The Earth shadow is modeled using the shadow $(\psi)$ function introduced by Ferraz Mello: $\psi$ equal zero when the satellite is in the shadow region and equal one when it is illuminated by the Sun. The drag components are given by Vilhena of Moraes based on the TD-88 temospheric model. The Hori's method for non-canonical systems is applied to solve the equations of motion. Algebric manipulator software is fundamental to do the necessary calculations. Secular and periodic effects on the orbital motion of artificial satellites are analyzed. Emphasis is given to the coupling terms that appear in the solution of the differential equations system. Using orbital data of the satellite CBERS-1 a study is done to analyze the order of magnitude of the variation of the semimajor axis due to the considered perturbations.

Key-words: Perturbation Theory, Atmospheric Drag, Direct Solar Radiation Pressure, Artificial Satellites.

\section{INTRODUÇÃ̃O}

A determinação precisa da órbita para satélites artificiais é um dos principais de-

\footnotetext{
*Endereço Eletrônico: jeanf eg@gmail.com

${ }^{\dagger}$ Endereço Eletrônico: rodolpho@feg.unesp.br
}

safios da tecnologia aeroespacial. Com o avanço do desenvolvimento da tecnologia espacial ouve a necessidade de melhorar a precisão do cálculo de órbita de satélites artificiais incluindo várias perturbações gravitacionais e não-gravitacionais. Neste artigo, estudamos o movimento translacional de um satélite artificial sujeito à ação do geopotencial, pressão de 
radiação solar direta (considerando a sombra da Terra) e arrasto atmosférico.

Encontramos na literatura diversos trabalhos que consideram efeitos perturbadores sobre satélites artificiais. Entre eles podemos citar: a) Em Brouwer e Hori [1] é apresentada uma solução analítica para o movimento de um satélite artificial no campo gravitacional terrestre considerando-se o efeito do arrasto atmosférico. A solução dada nesse trabalho apresenta termos espúrios de Poisson (função periódica do tempo multiplicada por uma função linear do tempo) que foram eliminados posteriormente por [2]; b) Segan [3] faz uma abordagem analítica levando em conta a influência do arrasto atmosférico sobre os elementos orbitais do satélite; c) Em [4, 5] é desenvolvida uma teoria para estudar os efeitos da pressão de radiação solar direta e o achatamento da Terra considerando um potencial com correções relativísticas para um satélite artificial da Terra; d) Em [6] os termos de ressonâncias produzidos pelos efeitos da pressão de radiação solar direta no movimento de um satélite artificial no campo gravitacional da Terra são analisados; e) Em [7] é desenvolvida uma teoria considerando o arrasto atmosférico até segunda ordem baseado no modelo atmosférico TD-88.

Quando são considerados os acoplamentos do geopotencial, pressão de radiação solar e arrasto atmosférico, as referências são pouco abrangentes na literatura. Por exemplo, em Vilhena de Moraes [8] é desenvolvida uma teoria de primeira ordem semi-analítica para estudar os efeitos da pressão de radiação solar direta e do arrasto atmosférico sobre o movimento orbital de satélites artificiais, com altura do perigeu entre 500 e $900 \mathrm{~km}$, considerando a sombra da Terra e incluindo os principais termos seculares devido ao achatamento da Terra.

Neste trabalho, são consideradas as seguintes perturbações: geopotencial, incluindo alta ordem e grau dos harmônicos $[9,10]$, a pressão de radiação solar direta, considerando a sombra da Terra [11], e o arrasto atmosférico, em que é utilizado para a densidade atmosférica o modelo TD-88 $[12,13]$. A abordagem analítica usada aqui para resolver as equações de movimento é o método de Lie-Hori [14, 15] para sistemas não-canônicos. A ação simultânea dos três efeitos deve ser considerada para satélites com altura do perigeu em torno de $700 \mathrm{~km}$ de altitude [8]. Esse é o caso de alguns dos satélites controlados pelo Centro de Controle de Satélite do INPE (Instituto Nacional de Pesquisa Espacial).

\section{GEOPOTENCIAL}

Apresentamos o formalismo hamiltoniano nas variáveis canônicas de Delaunay:

$$
\begin{aligned}
L & =\sqrt{\mu a}, \quad G=L \sqrt{1-e^{2}}, \quad H=G \cos i, \\
& \mu(=G M),
\end{aligned}
$$

$\mu$ é o parâmetro gravitacional da Terra $(\approx$ $\left.3,986 \times 10^{5} \mathrm{~km}^{3} / \mathrm{s}^{2}\right)$, a é o semi-eixo maior, $e$, a excentricidade, $i$ a inclinação, $l=M$, a anomalia média, $g=\omega$, o argumento do perigeu e $h=\Omega$, a longitude do nodo ascendente. A expressão analítica para o geopotencial expresso nas variáveis de Delaunay é dada por [9]:

$$
\begin{aligned}
U & =\frac{\mu}{r}+\frac{\mu}{L^{2}} \sum_{m=0}^{N} \sum_{k=-N}^{N} \sum_{q=-Q}^{Q} \gamma_{k m}\left(\bar{C}_{m}^{k q} \cos \Psi\right. \\
& \left.+\bar{S}_{m}^{k q} \operatorname{sen} \Psi\right)
\end{aligned}
$$

com

$$
\begin{aligned}
\Psi & =k g+(k+q) l+m(h-\theta)+(k-m) \frac{\pi}{2}, \\
\bar{C}_{m}^{k q} & =\sum_{j=j_{1}}^{N} \bar{Q}_{j m}^{k q} \bar{C}_{j m}, \\
\bar{S}_{m}^{k q} & =\sum_{j=j_{1}}^{N} \bar{Q}_{j m}^{k q} \bar{S}_{j m}, \\
\bar{Q}_{j m}^{k q} & =\left(\frac{\mu}{L^{2}}\right)^{j} \bar{A}_{j m}^{k}(i) G_{j,(j-k) / 2, q}(e), \\
\gamma_{k m} & =(-1)^{E((k-m+1) / 2)}, \\
j_{1} & =\max \left[k_{1}, k_{1}+2 E\left(\left(m+k_{1}+1\right) / 2\right)\right], \\
k_{1} & =|k|+2\left(\delta_{0 k}+\delta 1 k\right),
\end{aligned}
$$

onde $\bar{C}_{m}^{k q}$ e $\bar{S}_{m}^{k q}$ são os coeficientes harmônicos 
do geopotencial normalizado, $\bar{A}_{j m}^{k}(I)=$ $\bar{F}_{j, m(j-k) / 2}(i)$ é a função da inclinação normalizado [16], $G_{j,(j-k) / 2, q}$ é a função da excentricidade [16], $N$ é a máxima ordem e grau dos coeficientes, levando em conta que $Q$ é o máximo valor do índice $q$. O símbolo $\sum^{\star}$ representa a adição com um passo 2 .

A hamiltoniana pode ser posta na forma:

$$
\begin{aligned}
H & =\frac{\mu^{2}}{2 L^{2}}+\frac{\mu^{2}}{L^{2}} \sum_{m=0}^{N} \sum_{k=-N}^{N} \sum_{q=-Q}^{Q} \gamma_{k m}\left(\bar{C}_{m}^{k q} \cos \Psi\right. \\
& \left.+\bar{S}_{m}^{k q} \operatorname{sen} \Psi\right)
\end{aligned}
$$

em que $\frac{\mu^{2}}{2 L^{2}}$ é o termo devido ao potencial não perturbado.

\section{PERTURBAÇÕES DEVIDAS AO ARRASTO ATMOSFÉRICO}

Satélites artificiais orbitando uma atmosfera planetária são submetidos ao arrasto atmosférico. Como a densidade atmosférica da Terra diminui com a altura, a órbita é mais afetada no perigeu (ou seja, próxima da superfície da Terra). Esta força causa perturbações seculares nos elementos orbitais diminuindo o semieixo maior e a excentricidade orbital, com isso, a órbita contrai-se e torna-se quase circular levando o satélite a colidir com a Terra. O arrasto atmosférico é a força responsável pelo tempo de vida útil de satélites artificiais [17, 18]. O módulo da expressão da aceleração do arrasto é dado por [17]:

$$
D=\frac{1}{2} \rho_{d} C_{D} \frac{S}{m} V^{2},
$$

em que $\rho_{d}$ é a densidade local do ar que depende da posição e do tempo, $C_{D}$ é o coeficiente de arrasto, $S$ é a área efetiva, $m$ é a massa do satélite, $V$ é a velocidade do satélite em relação à atmosfera da Terra. A densidade $\rho_{d}$ da atmosfera depende de vários fatores, entre eles as variações de temperatura nas camadas atmosféricas de acordo com o ciclo solar de onze anos, as variações com a mudança diária na atividade na superfície solar, a variação diurna, as variações com atividade geomagnética, as variações semi-anuais, e as variações latitudinais e sazonais na termosfera baixa. O coeficiente de arrasto $C_{D}$ é função de parâmetros que dependem de propriedades da superfície do satélite (material, acabamento e temperatura), do ângulo de incidência, temperatura e velocidade das moléculas, etc. A área efetiva $S$ é determinada pela configuração e o tamanho do veículo, juntamente com o ângulo de ataque em relação ao fluxo atmosférico. Esta superfície é conhecida como área projetada pois está associada com a área externa do satélite projetada na direção da velocidade relativa com a atmosfera.

Vários modelos analíticos e numéricos para a densidade $\left(\rho_{d}\right)$ e para o coeficiente de arrasto $\left(C_{D}\right)$ podem ser encontrados na literatura $[19,20]$. Neste trabalho, será usado o modelo atmosférico TD-88 que é válido para altitudes entre 150 a $1200 \mathrm{~km}[12,13]$. O modelo TD88 além de ser simples conduz resultados comparáveis com outros modelos mais sofisticados quando usado em teorias de satélite artificiais [21]. O modelo TD-88 é bastante utilizado em teorias analíticas $[3,7,22]$.

A densidade $\rho_{d}$ pode ser reescrita na seguinte forma [22]:

$$
\begin{aligned}
\rho_{d} & =f_{x} f_{0} k_{0} \times \\
& \times\left\{\sum_{n=1}^{7} K_{n, 0} \sum_{k=0}^{\infty}\left(G_{n k} \cos k E+G_{n k}^{\prime} \operatorname{sen} k E\right)\right. \\
& +\sum_{\epsilon, \delta} \sum_{n=1}^{7} \sum_{j=1}^{3} K_{n, j} A_{j}\left(1+\frac{c_{j}^{2}}{4}\right) \sum_{m=0}^{\infty} F_{m}\left(\beta_{1}\right) \times \\
& \times \sum_{k=0}^{\infty}\left[G_{n k} \cos (k+\epsilon m) E+G_{n k}^{\prime} \operatorname{sen}(k+\epsilon m) E\right] \\
& +\sum_{\epsilon, \delta} \sum_{n=1}^{7} \sum_{j=1}^{3} K_{n, j} \frac{c_{j}}{2} \sum_{m=0}^{\infty} F_{m}\left(\beta_{j}\right) \times \\
& \times \sum_{k, k^{\prime}=0}^{\infty}\left[\left(G_{n k} A_{k^{\prime} j}+G_{n k}^{\prime} B_{k^{\prime} j}\right) \cos \left(k+\delta k^{\prime} \delta \epsilon m\right) E\right. \\
& \left.\left.+\left(G_{n k} A_{k^{\prime} j}+G_{n k}^{\prime} B_{k^{\prime} j}\right) \operatorname{sen}\left(k+\delta k^{\prime} \delta \epsilon m\right) E\right]\right\}
\end{aligned}
$$


em que $E$ é a anomalia excêntrica, $\delta$ e $\epsilon$ podem ser \pm 1 e:

$$
\begin{aligned}
& A_{k^{\prime} j}=\gamma_{2 k^{\prime}} \cos \omega+\frac{1}{4} c_{j} \cos 4 \omega \\
& B_{k^{\prime} j}=\sigma_{2 k^{\prime}} \operatorname{sen} 2 \omega+\frac{1}{4} \sigma_{4 k^{\prime}} \operatorname{sen} 4 \omega .
\end{aligned}
$$

A equação (4) pode ser expressa em termos da anomalia média e do tempo local. Se os termos periódicos não são considerados, temos:

$$
\begin{aligned}
\rho_{s} & =f_{x} f_{0} k_{0} \sum_{n=1}^{7} G_{n, 0}\left\{K_{n, 0}+\sum_{j=1}^{3} K_{n, j} A_{j} \times\right. \\
& \left.\times\left[\left(1+\frac{c_{j}^{2}}{4}\right)+\frac{c_{j}}{4} A_{0, j}\right]\right\},
\end{aligned}
$$

onde $G_{n, 0}$ e $A_{0, j}$ são funções da inclinação, e $\rho_{s}$ é a parte secular devida à densidade atmosférica.

\section{PRESSÃO DA RADIAÇÃO SOLAR}

A pressão de radiação solar é gerada através do contínuo fluxo de fótons que se chocam com a superfície do satélite em que pode absorver ou refletir este fluxo. A taxa da quantidade de movimento de todos os fótons incidentes na superfície do satélite origina a força de radiação solar. Esta perturbação pode causar variações nos elementos orbitais do satélite. É grande a sua influência nos satélites geoestacionários (aproximadamente $36000 \mathrm{~km}$ de altitude).

O interesse sobre a influência da perturbação devido a pressão de radiação solar no movimento de satélites artificiais teve inicio no princípio da década de 60 para explicar a discrepância entre teoria e observação de órbitas de satélites do tipo balão [23-26].

A aceleração causada pela pressão de radiação atua na direção Sol-satélite, no sentido oposto ao versor Terra-Sol, $\left(\hat{r}_{s}\right)$, é dada por [11]:

$$
\vec{A}_{P R}=-\nu C_{R} \frac{S}{m} P_{s} \hat{r}_{s}
$$

com $\nu$ sendo o fator de eclipse, que vale 0 quando o satélite se encontra na sombra da Terra e 1 quando o satélite está iluminado, $C_{R}$ é um fator que depende da refletividade do satélite, denominado de coeficiente de pressão de radiação, $S$ é a secção transversal quando observada na direção de incidência dos raios solares e $m$ a massa do satélite. $P_{S}$ é a pressão de radiação na órbita terrestre, e vale aproximadamente $4,55 \times 10^{-6} \mathrm{~N} / \mathrm{m}^{2}, \hat{r}_{s}$ é o raio vetor do Sol relativo à Terra.

Considerando um sistema geocêntrico cujo plano fundamental é o terminador terrestre, a função de forças devido à radiação solar é dada por [11]:

$$
R=\rho r \cos \lambda
$$

com

$$
\rho=k \frac{S}{m} q,
$$

onde $r$ é a distância geocêntrica do satélite, $\lambda$ é o ângulo definido pela direção geocêntrica do satélite e o pólo escuro do terminador terrestre. Sendo $k$ uma constante que depende do mecanismo de reflexão da luz na superfície do satélite, $S$ a área da secção transversal, $m$ a massa do satélite, e $q$ a pressão de radiação à distância da órbita da Terra dada por:

$$
q=4,65 \times 10^{-5} \mathrm{~g} \cdot \mathrm{cm} / \mathrm{s}^{2} .
$$

O ângulo pode ser expresso por [11]:

$$
\cos \lambda=\sum_{i=1}^{6} A_{i} \cos (f+\lambda),
$$

com $A_{i}(i=1, \ldots, 6)$ são funções da inclinação do plano da órbita e obliqüidade da eclíptica. $f$ é a anomalia verdadeira e os $\lambda_{i},(i=1, \ldots, 6)$, são os ângulos que dependem do argumento do perigeu $\omega$, da longitude do nodo ascendente $\Omega$ e da longitude do Sol, $\Theta$.

Para levar em conta a influência da sombra da Terra, temos que introduzir a função sombra $\psi$ [11]. Esta função é igual a 1 se o satélite está iluminado e igual a 0 se o satélite está na sombra. 
Como $\psi$ é uma função do ângulo $\lambda$, podemos expandir $\psi$ em uma série de Fourier dada por [27]:

$$
\begin{aligned}
\phi & =\operatorname{arcsen} \frac{R_{T}}{r}=\operatorname{arcsen} \frac{R_{T}(1+e \cos f)}{a\left(1-e^{2}\right)} \\
& =\sum \phi_{n}\left(a, R_{T}, e\right) \cos n f, \\
\psi & =1-\frac{\phi}{\pi}-\sum \frac{2}{p \pi} \operatorname{sen} p \phi \cos p \lambda, \\
\cos \psi & =\left[1-\frac{R_{T}^{2}(1+e \cos f)^{2}}{a^{2}\left(1-e^{2}\right)}\right]^{1 / 2} \\
& =\sum C_{n}\left(a, R_{T}, e\right) \cos n f, \\
\operatorname{sen} \psi & =\frac{R_{T}}{a\left(1-e^{2}\right)}(1+e \cos f) .
\end{aligned}
$$

em que $R_{T}$ é o raio médio da Terra.

\section{EQUAÇÕES DE MOVIMENTO}

Usando as variáveis de Delaunay $\left(L_{1}=\right.$ $\left.L, L_{2}=G, L_{3}=H, l_{1}=l, l=g, l_{3}=h\right)$, as equações de movimento de um satélite artificial submetido a perturbações do geopotencial, pressão de radiação solar direta (incluindo efeitos da sombra) e o arrasto atmosférico podem ser postas na seguinte forma [27]:

$$
\begin{aligned}
\frac{d L_{i}}{d t} & =\frac{\partial H}{\partial L_{i}}+\psi \frac{\partial R}{\partial l i}+P_{i}=Z_{i}\left(L_{i}, l_{i}\right) \\
\frac{d l_{i}}{d t} & =-\frac{\partial H}{\partial L_{i}}-\psi \frac{\partial R}{\partial l i}-Q_{i}=\bar{Z}_{i}\left(L_{i}, l_{i}\right)
\end{aligned}
$$

com $(i, j=1,2,3)$ e $H$ sendo a hamiltoniana devido ao geopotencial, $R$ a função força devido a pressão de radiação solar, $\psi$ é a função sombra da Terra e $P_{i}$ e $Q_{i}$ as componentes do arrasto.

Para os modelos considerados do arrasto at- mosférico e da pressão de radiação solar, temos:

$$
\begin{aligned}
P_{i} & =-A \rho_{d} \sum_{j} p_{i}^{j}\left(L_{1}, L_{1}, L_{1}\right) \times \\
& \times \cos \left(\alpha_{j} l_{1}+\beta_{j} l_{2}+\gamma_{j} l_{3}\right), \\
Q_{i} & =-A \rho_{d} \sum_{j} q_{i}^{j}\left(L_{1}, L_{1}, L_{1}\right) \times \\
& \times \operatorname{sen}\left(\alpha_{j} l_{1}+\beta_{j} l_{2}+\gamma_{j} l_{3}\right),
\end{aligned}
$$

com

$$
A=\frac{1}{2} \frac{S C_{D}}{m}
$$

e

$$
\psi \frac{\partial R}{\partial l_{j}}=\sum \rho B_{L_{j}} \operatorname{sen}\left(\alpha l_{1}+\beta l_{2}+\gamma l_{3}+\delta \Theta\right),
$$

$$
\begin{aligned}
\psi \frac{\partial R}{\partial L_{j}} & =-\rho a_{L_{j}} \\
& -\sum \rho b_{L_{j}} \cos \left(\alpha l_{1}+\beta l_{2}+\gamma l_{3}+\delta \Theta\right)
\end{aligned}
$$

onde $B_{l}, a_{L}$ e $b_{L}$ são funções de $L_{1}, L_{2}$ e $L_{3}, P_{i}$ e $Q_{i}$ são as componentes do arrasto [22].

O sistema de equações (9) pode ser resolvido aplicando o método de teoria de perturbação de Lie-Hori [14, 15] para sistemas nãocanônicos. A solução explícita para a parte secular até a segunda ordem por ser muito extensa não será exposta aqui, mas pode ser consultada na referência [28]. Esta solução é obtida considerando no núcleo integrável do método de Lie-Hori todos os termos seculares do geopotencial, como sugerido por [29], sendo que serão feitas aplicações com a solução secular de primeira e segunda ordem para um caso específico, considerando o satélite CBERS-1.

\section{A FORMA CONSIDERADA PARA A SOLUÇÃ̃O}

A solução geral pode ser representada como segue [28]: 
a) variáveis métricas (parte secular):

$$
\begin{aligned}
\left(L_{1}, L_{2}, L_{3}\right) & =\left(L_{10}, L_{20}, L_{30}\right)+(A+\mathcal{R} A) t \\
& +(A \rho) t,
\end{aligned}
$$

sendo $L_{10}, L_{20}, L_{30}$ constantes de integração devidas à solução de ordem zero enquanto que $\mathcal{R}$, $A$ e $\rho$ representam os termos seculares devido ao geopotencial, arrasto e a pressão de radiação solar, respectivamente. Os termos dos acoplamentos $\mathcal{R} A$ (geopotencial-arrasto) e $A \rho$ (arrasto-pressão de radiação) são termos de segunda ordem que surgem na resolução do problema quando aplica-se o método de Lie-Hori [14].

b) variáveis angulares (parte secular):

$$
\begin{aligned}
\left(l_{1}, l_{2}, l_{3}\right) & =\left(l_{10}, l_{20}, l_{30}\right)+\left(n_{l}, n_{g}, n_{h}\right) t+\rho t \\
& +(\mathcal{R}+\mathcal{R} \rho) t,
\end{aligned}
$$

sendo $\left(l_{10}, l_{20}, l_{30}\right)$ constantes de integração devidas a solução de ordem zero, $\left(n_{l}, n_{g}, n_{h}\right)$ são os movimentos médios de $l, g$ e $h$, respectivamente. Ademais, $\mathcal{R}, A$ e $\rho$ representam os termos seculares devidos ao geopotencial, arrasto e pressão de radiação solar, respectivamente. O termo de acoplamento $\mathcal{R} \rho$ (geopotencialpressão) é um termo de segunda ordem que surge na resolução do problema quando aplicase o método de Lie-Hori [14].

As funções geratrizes de primeira ordem são dadas explicitamente em [28]. Deve ser observado que a solução encontrada não apresenta termos espúrios de Poisson por conta do método de perturbação utilizado.

As simulações são feitas considerando apenas os termos devido ao $J_{2}$ (achatamento da Terra) para o geopotencial e alguns termos do arrasto e da pressão. As equações utilizadas para calcular a variação $(\mathcal{D} a)$ do semieixo maior podem ser escritas na forma a seguir (parte secular):

Ordem um (arrasto atmosférico):

$$
\delta a=-1728 \times 10^{5} \frac{A \mu \rho_{s} a}{L} t .
$$

O modelo conduziu a não-existência de perturbações seculares puras devidas ao geopotencial e à pressão de radiação solar direta no semi- eixo maior, que está de acordo com [8, 11, 27].

Ordem dois (arrasto - geopotencial):

$$
\begin{aligned}
\delta a & =\left[\sqrt { L ^ { 2 } - G ^ { 2 } } \left(\mu^{2} R_{T}^{2}\left(G^{2}-3 H^{2}\right) J_{2}\right.\right. \\
& \left.\left.-(4 / 3) G^{5} L\right)^{2} L^{7}\right]\left[2916 \times 10^{5} G^{3} e J_{2} a A \mu^{3} \times\right. \\
& \times\left(\mu^{2} R_{T}^{2} L^{3} J_{2}\left(G^{2}-3 H^{2}\right) \sqrt{L^{2}-G^{2}}\right. \\
& +(3 / 4)\left(\mu^{2} R_{T}^{2}\left(G^{2}-3 H^{2}\right) J_{2}-(4 / 3) G^{5} L\right) \times \\
& \left.\times\left((8 / 9) L^{4}-G^{2} L^{2}+G^{4}\right)\right)\left(G^{2}-3 H^{2}\right) R_{T}^{2} \times \\
& \left.\times\left((8 / 9)+e^{2}\right) \rho_{s}\right] .
\end{aligned}
$$

Ordem dois (arrasto - pressão):

$$
\begin{aligned}
\delta a & =0,04589 A \rho_{s} L^{4} G^{5} a \mu^{-2}\left[-4 G^{5} L\right. \\
& \left.+3 \mu^{2} J_{2} R_{T}^{2} G^{2}-9 \mu^{2} J_{2} R_{T}^{2} H^{2}\right]
\end{aligned}
$$

onde $t$ é o tempo em dias. A solução geral não será exposta por ser muito extensa.

\section{SIMULAÇÃO}

Algumas simulações foram feitas usando os seguintes dados do satélite CBERS-1 (http://www.cbers.inpe.br, [30]),

$$
\begin{array}{lr}
a=7148,948 \mathrm{~km} & e=0,00001 \\
i=98,406^{\circ} & \rho_{s}=1,72502 \times 10^{-10} \mathrm{~kg} / \mathrm{m}^{3} \\
C_{D}=2,62 & S=15,37 \mathrm{~m}^{2} \\
A=0,01342 \mathrm{~m}^{2} / \mathrm{kg} & C=1,44 \\
\nu=1 & m=1550 \mathrm{~kg} .
\end{array}
$$

Calculando a variação para o semi-eixo maior, obtemos [28]:

a) solução de primeira ordem usando a equação (16):

$$
\delta a=-2,13541 \times 10^{-3} \mathrm{~km} / \text { dia } .
$$

b) solução de segunda ordem devido ao acoplamento do arrasto com o geopotencial equação (17):

$\delta a=-6,89932 \times 10^{-7} \mathrm{~km} / \mathrm{dia}$.

c) solução de segunda ordem devido ao acoplamento do arrasto com a pressão de 
radiação equação (18):

$$
\delta a=-1,18190 \times 10^{-11} \mathrm{~km} / \mathrm{dia} .
$$

Usando os dados do satélite CBERS-1 a perturbação devido ao arrasto atmosférico é calculada usando a equação (3), temos:

$$
D=-1,29076 \times 10^{-8} \mathrm{~m} / \mathrm{s}^{2} .
$$

A aceleração devido a pressão de radiação é calculada usando a equação (6), temos:

$$
\rho=6,63984 \times 10^{-8} \mathrm{~m} / \mathrm{s}^{2} .
$$

Deve ser observado que o produto das equações (19) e (20) dá uma perturbação de acoplamento do arrasto com a pressão de magnitude da ordem de $10^{-16} \mathrm{~m} / \mathrm{s}^{2}$, porém, a solução analítica obtida dá para este satélite um termo do efeito do acoplamento da ordem de $10^{-11} \mathrm{~m} / \mathrm{s}^{2}$. Esta queda da ordem de grandeza é devido aos termos que vêm dos movimentos médios que estão presentes nos denominadoros das expressões analíticas para os termos de acoplamentos [28].

\begin{tabular}{cl}
\hline Altitude $\mathrm{h}(\mathrm{km})$ & $\rho / D$ \\
200 & 0,00029 \\
300 & 0,00540 \\
400 & 0,04155 \\
500 & 0,17836 \\
600 & 0,17836 \\
700 & 2,20225 \\
800 & 7,27311 \\
\hline
\end{tabular}

TABELA I: Relação entre a magnitude das perturbações devido a pressão de radiação solar e arrasto.

O quociente da equação (19) e (20) dá $|\rho / D|$, ou seja, para a altitude do satélite CBERS-1 a magnitude da perturbação devido a pressão de radiação solar é maior que a perturbação devido ao arrasto. A Tabela-1 representa esta relação para órbitas circulares e para algumas altitudes particulares. A densidade atmosférica $\rho_{d}$ é calculada usando o modelo TD88.

A Figura 1 representa a variação do semieixo maior levando em conta o efeito do arrasto atmosférico para um período de 100 dias. A variação da densidade é calculada usando o modelo atmosférico TD-88.

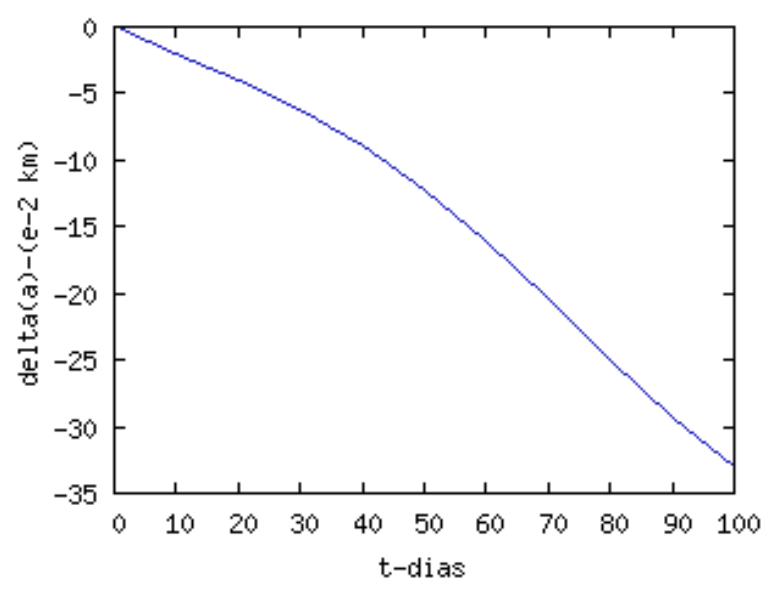

FIGURA 1: Variação do semi-eixo maior devido ao arrasto atmosférico.

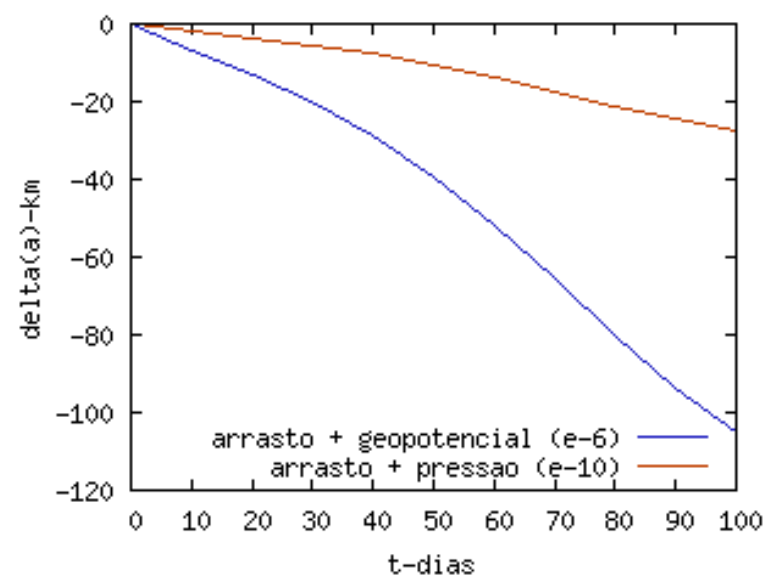

FIGURA 2: Variação do semi-eixo maior devido acoplamento do arrasto-geopotencial e do arrastopressão de radiação.

A Figura 2 mostra que para o satélite CBERS-1, a influência do acoplamento da perturbação do arrasto-geopotencial é mais significante do que o acoplamento da perturbação do arrasto-pressão de radiação. Isto pode ser explicado pela baixa razão área/massa do satélite 
CBERS-1. Para satélites com grande razão área/massa a influência da pressão de radiação é mais acentuada.

\section{CONCLUSÕES}

Os efeitos combinados das perturbações causadas pelo geopotencial, pressão de radiação solar direta (considerando a sombra da Terra) e o arrasto atmosférico (usando o modelo TD-88) são estudados. As equações de movimento (expressas em termos das variáveis de Delaunay) são integradas aplicando o método de Lie-Hori analiticamente para sistemas não-canônicos.

Foram feitas simulações usando dados do satélite CBERS-1. Na solução de primeira ordem do método de Lie-Hori é encontrada uma redução do semi-eixo maior devido ao arrasto cerca de $10^{-3} \mathrm{~km} /$ dia. Para a solução de segunda ordem é considerado para o semieixo maior, termos de perturbações acoplados: geopotencial-arrasto (diminuindo cerca de $10^{-7}$ $\mathrm{km} /$ dia) e pressão de radiação solar-arrasto (diminuindo cerca de $10^{-11} \mathrm{~km} /$ dia). Estes resultados podem ser importantes para missões que necessitem da determinação de órbita precisa já que é requerido que as órbitas dos satélites sejam determinadas com grande precisão, para que, a cada instante, a posição do satélite possa ser prevista. Em qualquer missão espacial, quer se exija que a órbita seja conhecida com maior ou menor precisão, o co- nhecimento do comportamento da órbita é vital para o sucesso da missão. Portanto, a ordem de grandeza com que cada perturbação afeta os elementos orbitais é de fundamental importância para os satélites artificiais. Principalmente para satélites com grande razão área/massa o efeito do acoplamento da pressão de radiação e o geopotencial são muito importantes.

Mostramos que a ordem do acoplamento da perturbação do arrasto com a pressão de radiação, não é simplesmente a multiplicação da ordem de ambas as perturbações. A multiplicação daria uma ordem de grandeza de $10^{-16} \mathrm{~km} /$ dia e ao utilizarmos a teoria encontramos, para os termos acoplados uma variação da ordem de $10^{-11} \mathrm{~km} /$ dia. Esta queda na ordem de grandeza é devido aos termos do movimento médio os quais, devido ao processo de integração, aparecem no denominador das expressões de acoplamento.

A aplicação do método de Lie-Hori é conveniente desde que nos permite calcular diretamente a variação dos elementos orbitais e analisar seu comportamento: secular, periódico de curto e longo período.

\section{Agradecimentos}

O primeiro autor agradece a CAPES pelo apoio financeiro. $\mathrm{O}$ segundo autor agradece a CAPES.
[1] D. Brouwer, G. Hori Theoretical evaluation of atmospheric drag effects in the motion of an artificial satellite. Astro. J. 66, 193 (1961).

[2] M.T.R.Fitzgibbon, R. Vilhena de Moraes, An analytical theory on the disturbed motion of an artificial earth satellite. The Journal of Astronautical Sciences 35, (2) 235 (1987).

[3] S. Segan, Analytical computation of atmospheric drag effects. Cel. Mech. 41, 381 (1988).

[4] M.I. El-Saftawy, A.A. El-Enna, The relativistic and direct solar radiation pressure effects on an axially symmetric spacecraft. I. The Hamiltonian and the canonical equations of motion. Appl. Math. Comput. 132, 505
(2002).

[5] A.A. El-Enna, M.I. El-Saftawy, The joint effect of relativistic and direct solar radiation pressure on an axially symmetric spacecraft. II. The solution and the elements of transformation. Appl. Math. Comput. 138, 247 (2003).

[6] F.A.Abd. El-Salam, L. Sehnal, A second order analytical atmospheric drag theory based on the TD-88 thermospheric density model. Cel. Mech. 90, 361 (2004).

[7] M.I. El-Saftawy, Analytical of the Ressonance Caused by Solar Radiation Pressure on a Spacecraft. Astrophysics and Space Science 
295, 407 (2004).

[8] R. Vilhena de Moraes, Combined Solar Radiation Pressure and Drag Effects on the Orbits of Artificial Satellites. Cel. Mech. 25, 281 (1981).

[9] E. Wnuk, Tesseral Harmonic Perturbations for High Order and Degree Harmonics. Cel. Mech. 44, 179 (1988).

[10] E. Wnuk, Harmonic Perturbations in the Keplerian Orbital Elements. ACTA Astronomica 40, 191 (1990).

[11] S. Ferraz Mello, Action de la Pression de Radiation Sur le Mouvement d'un Satellite Artificial de la Terre. In: Proc. Intern. Astronaut. Congress, 14th, Vol. IV, 1963. Warsaw: PWN (1965).

[12] L. Sehnal, Thermospheric Total Density Model TD. Bull. Astron. Inst. Czechosl. 39, 120 (1988).

[13] A. Bezddek, D. Vokrouhlicky, Semi-Analytic Theory of Motion for Close-Earth Spherical Satellites Including Drag and Gravitational Perturbations. Planetary and Science 52, 1233 (2004).

[14] G.I. Hori, Theory of General Perturbation for Non-Canonical Systems. Publ. Astr. Soc. Japan. 23, 567 (1971).

[15] S. Ferraz Mello, Canonical Perturbation Theories. New York: Springer (2007).

[16] W.M. Kaula, Theory of Satellite Geodesy. Walthan: Bleisdell (1966).

[17] D.G. King-Hele, Theory of Satellite Orbits in an Atmosphere. London: Butter Worths (1964).

[18] J. Lafontaine, R. Mamen, Orbit Lifetime Prediction and Safety Considerations. IAF Paper. 84, (1984).

[19] R. Vilhena de Moraes, Non-Gravitational Disturbing Forces. Adv. Space Res. 14, (5) 45 (1994).

[20] T.R. Silva, Cálculo de Coeficientes de Arrasto para Satélites Artificiais. Dissertação
(Mestrado) - FEG-UNESP, Guaratinguetá (2001).

[21] G.W. Nicoletti, R. Vilhena de Moraes, Estudo Comparativo de Modelos Analíticos para Densidade Atmosférica. In: Resumos do CBDO 49, (2000).

[22] R. Vilhena de Moraes, Analytical Computation of Drag Perturbations using thermospheric Model TD-88, in Orbital Dynamics of Natural and Artificial Objects. In: Brazilian Colloquium on Orbital Dynamics, 135 (1989).

[23] P. Musen, The Influence of the Solar Radiation Pressure on the Motion of an Artificial Satellite. Geophys. Res. 65, (5) (1960).

[24] Musen, P., Bryant, R.; Bailie, A. Perturbations in Perigee Height of Vanguard I. Science 131, 935 (1960).

[25] Shapiro, I. I., Jones, H. M. Perturbations of the Orbit of the Echo Balloon. Science 132, 1484 (1960).

[26] P.E. Zadunaisky, I.I. Shapiro, H.M. Jones, Experimental and Theoretical Results on the Orbit of Echo I. SAO SP. RESP. 61, (1961).

[27] R. Vilhena de Moraes, Ação da pressão de radiação solar e do arrasto atmosférico sobre órbitas de satélites artificiais. Tese (Doutorado) - Instituto Tecnológico da Aeronáutica, São José dos Campos (1978).

[28] J.P.S. Carvalho, Ação de Forças Gravitacionais e Não Gravitacionais sobre o Movimento Orbital de Satélites Artificiais. Dissertação (Mestrado) - FEG-UNESP, Guaratinguetá (2007).

[29] Breiter, S. Second-Order Solution for the Zonal Problem of Satellite Theory. Cel. Mech. 67, 237 (1997).

[30] H.K. Kuga, R.K. Rao, V. Carrara, Satélites Artificiais: Movimento Orbital. São José dos Campos: INPE (2000). 